\title{
Prediction and Prevention System for Sticking Type Breakout in Continuous Casting*
}

\section{Introduction}

Increase in casting speed for enhanced productivity in continuous casting plant often causes a sticking type breakout because of poor lubrication, melt penetration into corner gap in mold and so on. Ratio of the sticking type breakout to the total number of breakout is beyond $60 \%$. Damage due to the breakout is serious.

There are a few reports on the technologies for breakout prediction. It is needed in earnest to develop a simple and reliable system for preventing the breakout. Kawasaki Steel Corp. has developed a prediction and prevention system for sticking type breakout with high reliability and easy maintenance.

\section{Principle and Characteristics of the System}

The principle of the prediction is based on measurement of the temperature on a copper mold plate. The breakout is warned when elevation of temperature is detected at adjacent positions on a horizontal line of copper mold plate below meniscus. The temperature is measured by exchangeable type of sheath thermocouples fixed by a spring onto the back face of copper plate through hollow stud-bolts; thus no need to machine specially the copper mold plate or to separate the plate from back-up plate (cooling water box) for setting the thermocouples. Measuring part is protected from leakage of cooling water so that accurate measurement is ensured. The number and position of temperature measurements can be determined in terms of time lapse from prediction to breakout by using following parameters: the mold length; $L$, a pitch of the stud-bolts along the horizontal line; $w_{x}$, the distance from the measuring position to the mold exit; $l$, the angle of a constricted line of a shell stuck to the mold; $\beta$, and the descending speed of constricted line of the shell; $\alpha V c(\alpha$ : constant, $V c$ : casting speed).

Figure 1 shows a flow chart of the prediction and prevention system. The number of parameters for the prediction is basically one, which is an upper critical rate of temperature change, $\dot{\theta}_{c r}$, obtained

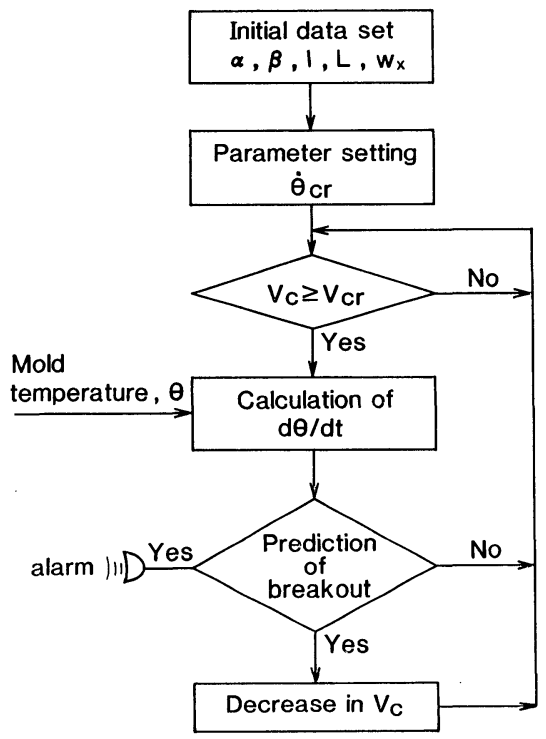

Fig. 1. Flow chart of sticking type breakout prediction and prevention system. from the changes in mold temperature at breakout. This means that the system has wide applicability to the other types of machines.

The operations of the system have shown that no breakout occurs without previous alarm and the frequency of false alarm with decreasing casting speed was less than twice in a month. No increase in the frequency of false alarm was observed in the case of special steel grade, such as steels accompanying $\delta-\gamma$ transformation during solidification.

Figure 2 shows an example of the changes in mold temperature when the breakout was predicted and prevented. It has been shown that the decrease in casting speed almost eliminates the breakout.

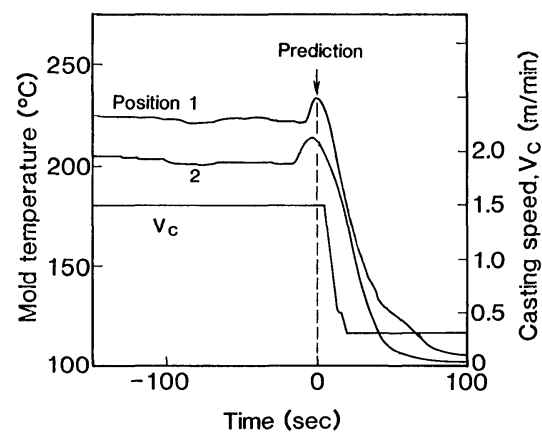

Fig. 2. Temperature changes in the mold when the breakout was predicted and prevented.

\footnotetext{
* For further information, write to Head Office, Kawasaki Steel Corporation, 2-3, Uchisaiwai-cho 2-chome, Chiyoda-ku, Tokyo 100. (C) 1988 ISIJ
} 